Maintenance Strategies: Decision Making Grid vs. Jack-Knife Diagram

Turuna Seecharan, University of Minnesota Duluth

Ashraf Labib, University of Portsmouth

Andrew Jardine, University of Toronto 


\title{
Maintenance Strategies: Decision Making Grid vs. Jack-Knife Diagram
}

\begin{abstract}
Purpose - Maintenance management is a vital strategic task given the increasing demand on sustained availability of machines. Machine performance depends primarily on frequency and downtime; therefore, ranking critical machines based on these two criteria is important to determine the appropriate maintenance strategy. This paper compares two methods, using case studies, to allocate maintenance strategies while prioritising performance based on frequency and downtime or Mean Time to Repair (MTTR): the Decision Making Grid (DMG) and Jack-Knife Diagram (JKD).
\end{abstract}

Design/methodology/approach - Literature indicates the need for an approach able to integrate maintenance performance and strategy in order to adapt existing data on equipment failures and to routinely adjust preventive measures. Maintenance strategies are incomparable; one strategy should not be applied to all machines, nor all strategies to the same machine.

Findings - Compared to the Pareto histogram, the DMG and JKD provide visual representations of the performance of the worst machines with respect to frequency and downtime, thus allowing maintenance technicians to apply the appropriate maintenance strategy. Each method has its own merits.

Originality/Value - Neither DMG nor JKD have been compared in the literature. Currently, the JKD has been used to rank machines, and the DMG has been used to determine maintenance strategies.

Research limitation/implication - This work compares only two methods based on their original conceptualisation. This is due to their similarities in using same input data and their main features. However, there is scope to compare to other methods or variations of these methods.

Practical application - This paper highlights how the DMG and JKD can be incorporated in industrial applications to allocate appropriate maintenance strategy and track machine performance over time.

Keywords - Decision Making Grid, Jack-Knife Diagram, Maintenance Management 
Paper Type - Research Paper 


\section{Introduction}

\subsection{Problem Statement}

The vast majority of maintenance models are aimed at answering efficiency questions, for example, "How can this particular machine be operated more efficiently?" They are not aimed at effectiveness, for example, "Which machine should we improve and how?" (Vanneste and van Wassenhove, 1995; Kobbacy et al., 1995; Cho and Parlar 1991). Yet practitioners are often interested in the latter question and are dissatisfied if a model is directly applied to an isolated problem. In an integrated approach to maintenance, efficiency analysis (do the thing right) should be preceded by effectiveness analysis (do the right thing). The Decision Making Grid (DMG) (Labib, 2004) and the Jack-Knife Diagram (JKD) (Knights, 2001) can work together to address this issue.

Maintenance costs and downtime depend on two factors: downtime frequency and downtime length or Mean Time To Repair (MTTR). Pareto histograms, traditionally used to prioritise failure codes, consider only one downtime criterion, typically MTTR, and are unable to analyse the two criteria simultaneously. In addition, according to Knights (2004), Pareto analysis has a number of deficiencies.

Firstly, as a Pareto histogram is usually based on downtime (or cost) alone, it cannot determine which factor(s) predominate in the downtime or costs associated with particular failure codes.

Secondly, Pareto histograms for failure codes ranked according to repair cost, equipment downtime, failure frequency and MTTR will generate four distinct lists of failure code priorities. A graphical means of simultaneously visualising equipment availability, reliability and maintainability problems as a function of equipment failure codes would be useful.

Thirdly, Pareto analysis of equipment downtime may not identify individual events with high associated downtimes or frequently occurring failures consuming relatively little downtime whilst causing frequent operational disturbances.

Fourthly, when there are many data to analyse, data stratification or hierarchical decomposition techniques are commonly used. There are two potential problems with the use of stratified Pareto analyses: (i) because hierarchical Pareto graphs are only prepared for the significant contributors of system downtime, failures associated with less significant components or functional failures 
are not explored; (ii) the same failure mode may appear in several of the lower level Pareto histograms.

In contrast, the DMG and JKD provide a single display that ranks failure codes according to the two important downtime factors, frequency of downtime and MTTR. Maintenance strategies are incomparable; that is, not all maintenance strategies are applicable to all failure codes. The DMG and JKD have the added advantage of allocating the appropriate maintenance strategy depending on the position of a machine according to these two downtime factors. In short, both provide a better means of establishing maintenance priorities.

DMG and JKD are alike in their reliance on mapping failure frequency and downtimes. Whilst the DMG acts as a map on which the performances of the worst machines are located according to multiple criteria, the JKD is a logarithmic scatterplot enabling failures to be classified according to acute or chronic characteristics. The object is to implement appropriate actions leading to the movement of machines towards improvement in these criteria (Labib, 2004).

\subsection{Performance Measures and Maintenance Strategies}

There is a gap between the literature and practice in understanding the effectiveness of maintenance performance measures and their impact on maintenance strategies and decision making. The majority of the literature promotes performance measures as a vital instrument that links strategies and management decisions with the execution of improvement initiatives (Neely, et al., 2005; Kaplan and Norton 1992). More specifically, in the area of maintenance, much literature emphasises the importance of maintenance performance measurements (Parida and Chattopadhyay, 2007; Simoes, et al., 2011; Kumar, et al., 2013).

Currently, the software packages for maintenance management are either called Computerised Maintenance Management Systems (CMMS) or Enterprise Resource Planning (ERP) systems with the current market leader being SAP software. CMMSs are dedicated to the management of maintenance in both corrective and preventive modes. ERP systems, formerly called Material Requisition Planning (MRPII), rely on using a unified database management system that integrates maintenance to other functions in the business; specifically, those related to logistics such as spare parts management. However, such systems usually run without making full use of the data from the CMMS/ERP system. In addition, such software packages tend to be used for 
data analysis and facilitating exchange of reports rather than for offering real decision support, see Labib (2003) for criticism of CMMSs.

There is an indication that when making decisions, maintenance managers tend not to rely on the key performance indicators and their data in the (CMMS). For example, empirical research by Muchiri et al (2010) casts doubt on the utility of maintenance performance measurement in terms of its impact on decisions taken. They find a relatively small number of decisions and changes are triggered by performance measurements. In fact, a significant number of maintenance managers are not satisfied with their performance measurements (Muchiri et al, 2010).

An early paper empirically investigating the link between maintenance strategies and performance finds a strong positive relationship between proactive and aggressive maintenance approaches (e.g. Total Productive Maintenance (TPM) or Reliability-Centred Maintenance (RCM)) and performance (Swanson, 2001). An empirical investigation of the relationship between business and maintenance strategies by Pinjala et al. (2006) says quality competitors tend to have more pro-active maintenance policies than others. In a survey of maintenance practices, Alsyouf (2009) concludes there is a need for more frequent adoption of such maintenance concepts as TPM and RCM.

In terms of selection of maintenance strategy, Labib et al. (1996) and Labib et al. (1997) suggest using the Multiple Criteria Decision Making (MCDM) technique of the Analytic Hierarchy Process (AHP). Bevilacqua and Braglia (2000) also advocate AHP. Al-Najjar and Alsyouf (2003) propose a conceptual model using fuzzy logic and multiple criteria to select cost effective maintenance approaches; Wang et al. (2007) suggest a model for the selection of maintenance strategies using the fuzzy variation of AHP. Oyarbide-Zubillaga et al. (2008) use discrete event simulation to optimise preventive maintenance; Picciolo et al. (2008) use Markov modelling to optimise periodic inspection. To select a maintenance strategy more generally, Bashiri et al. (2011) propose using a fuzzy linear assignment method, whilst Pourjavad and Shirouyehzad (2014) propose the fuzzy Analytic Network Process (ANP). In more specific analyses, Lazakis and Olcer (2015) advocate a fuzzy multiple attribute group approach to select a maintenance strategy in the maritime industry, and Nakhjavani et al. (2014) suggest a hybrid approach using both ANP and the Decision-Making Trial and Evaluation Laboratory (DEMATEL) method to select the optimal maintenance stategy in the pharmaceutical industry. 
The above literature indicates the need for an approach able to integrate maintenance performance and maintenance strategy, one able to adapt to existing data on equipment failures and to routinely adjust preventive measures.

The DMG and JKD models have received little attention in literature, yet they have the potential to routinely analyse data captured in CMMS and to aid decision makers in the implementation of performance-enhancing maintenance strategies. Their visual representation of machine performance is an appealing feature distinguishing them from other approaches. Their ability to prioritise machine downtime based on two criteria (frequency and length of downtime) has already been noted. In addition, their ability to classify maintenance strategies enables the allocation of the different responsibilities of preventive measures across machine groups. Such an approach is useful to the decision maker, first to plan the implementation of appropriate strategies and then to monitor their impact.

In the literature, ELECTRE-SORT is a sorting method able to consider an unlimited number of criteria in order to assign machines to incomparable strategies (Ishizaka and Nemery, 2014). The main drawback of ELECTRE-SORT is its complexity. In cases where a decision needs to be taken quickly, either the DMG or the JKD is sufficient. We limit the concept of maintenance to these two approaches because they are the most commonly used in industry, and they address both aspects of maintenance in terms of being system-based (RCM), or human-based (TPM)

\section{The Decision Making Grid (DMG)}

The DMG acts as a map on which the performances of the worst machines are located according to multiple criteria. The DMG model was initiated by Labib (1996). During subsequent research, it was integrated into CMMS by Labib (1998), into AHP by Labib et al. (1998a) and into fuzzy logic by Labib et al. (1998b). It has since become widely implemented in industry (Fernandez, et al., 2003; Labib, 2004; Burhanuddin, 2007; Tahir et al., 2008; Shahin and Attarpour, 2011; Aslam-Zainudeen and Labib, 2011; Tahir et al., 2014; Mc Leod et al., 2015).

In the DMG, assets are plotted on two separate dimensions: downtime and failure frequency. Downtime is commonly represented by the key performance indicator, MTTR, whilst failure frequency is often represented as Mean Time between Failures (MTBF); an increase in failure frequency equals an increase in the reciprocal of MTBF. Some argue the DMG is insufficient as 
the consideration of only two criteria does not necessarily result in a wise decision; it has been suggested, for instance, that cost should be included as well. However, cost as a dimension is already captured if we consider cost to be analogous to downtime, with both seen as measures of severity (Labib, 2014).

The concept of the DMG enables the user to determine when to apply TPM- or RCM-based approaches. Figure 1 is adapted from Labib (2004) who states: "The significance of this approach is that rather than treating RCM and TPM as two competing concepts it unifies them within a single analytical model".
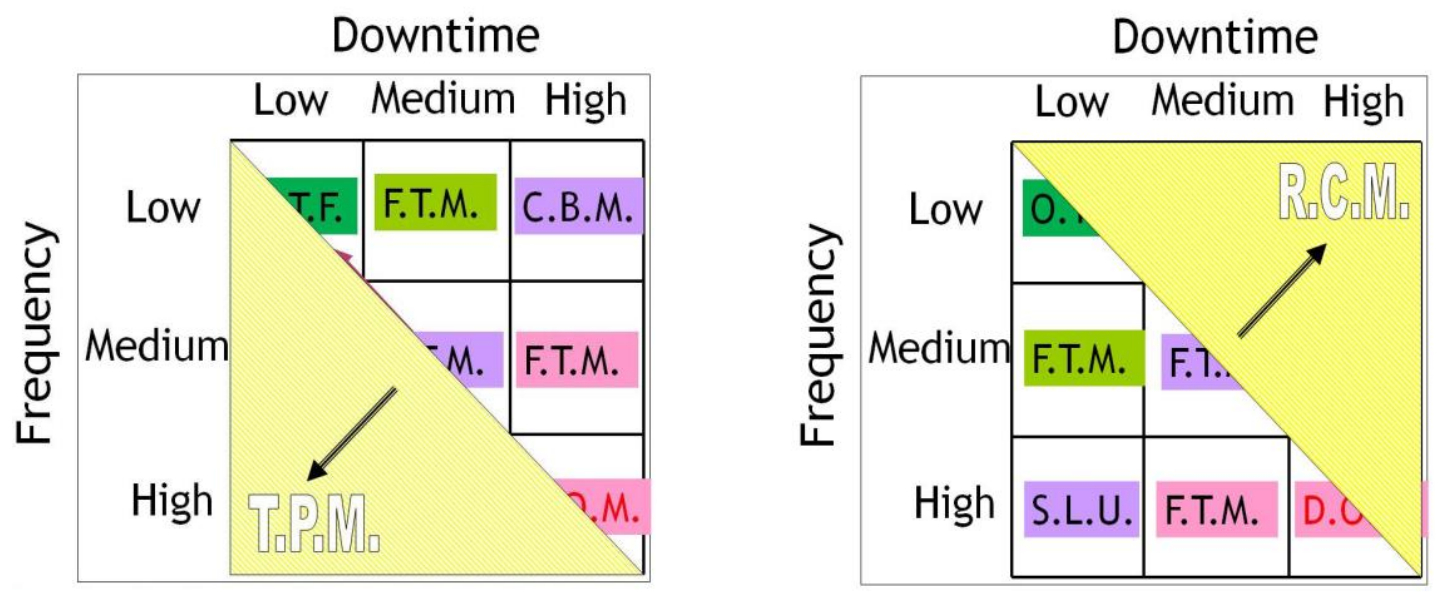

Figure 1: When to apply RCM and TPM in DMG (Labib, 2004)

The DMG comprises nine sections, each of which is represented by one of five different maintenance strategies. The asset's position on the grid determines the accompanying optimum maintenance strategy. Note that the DMG refers to preventive maintenance (PM) as Fixed Time Maintenance (FTM) instead. This is done on purpose in order to avoid confusion with TPM when referring to it in the DMG model. Note that CBM is used in the DMG to signifiy 'predictive maintenance', which is defined in the Maintenance Terminology Standards (BSI 13306) as "Condition based maintenance carried out following a forecast derived from the analysis and evaluation of the significant parameters of the degradation of the item". 


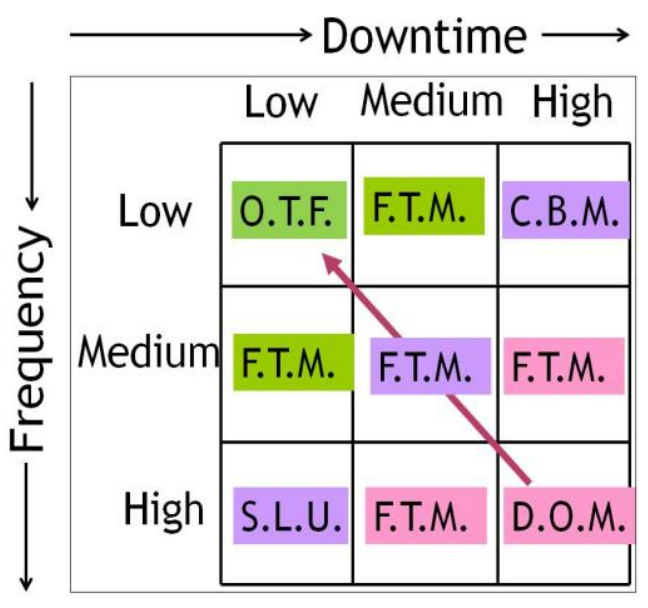

Figure 2: Decision Making Grid asset strategies (Labib 2004)

The objective is to implement actions leading to the movement of the asset's performance towards the top left section representing low failure frequency and low downtime (Labib, 2004). Contributions towards classifications using greater than two maintenance criteria include ELECTRE-SORT, developed by Ishizaka and Nemery (2014), group Dominance-Based Rough Set Approach (DRSA), developed by Chakhar, Ishizaka, Labib and Saad (2016), and application of DRSA in classification of maintenance spare parts (Hu et al, 2017). However, more research is needed to optimise the number of categories and demonstrate their practical applicability.

\subsection{DMG Strategies}

The allocation of maintenance strategies to various machines is analogous to prescribing drugs for different patients. In the field of medicine, two extremes may occur, i.e. prescribing one drug to all patients and prescribing all drugs to one patient, are neither feasible nor acceptable. The same applies in maintenance: one strategy should not be applied to all machines, nor all strategies to the same machine.

Five maintenance strategies are presented below; the strategy "prescribed" for a specific failure code depends on the failure's location in the DMG.

Operate to Failure (OTF) Assets falling in the top left-hand section of the grid represent machines with low failure frequency and low downtime. This is the optimal state and is the area of the grid towards which all asset performance figures should aspire. In this case, the suggested 
strategy, OTF, sustains the best practice for the machines concerned. Note that OTF does not imply ignoring the asset but rather to audit sustainability of the existing best practice.

Skills Level Upgrade (SLU) When breakdowns occur frequently but are fixed quickly resulting in little downtime, the SLU strategy should be used. One of the underpinning elements of TPM is the transfer of some basic maintenance skills from maintenance technicians to the front-line operators in production, hence the term "productive maintenance" (Nakajima, 1988; Hartmann, 1992; and Willmott, 1994). As mentioned earlier, chronic losses (“death by a thousand cuts") should be dealt with using TPM-type initiatives. For example, menial tasks such as machine resetting or minor adjustments can be carried out by operators once they have received the correct training, i.e. SLU. Another variation of SLU is to de-skill the maintenance function on a machine by focusing on improving its maintainability.

Condition Based Maintenance (CBM) Assets with low frequency of failure but high downtime when failures (infrequently) occur call for CBM. Such assets can have catastrophic failures resulting in extended downtime ("show stoppers"). By monitoring their condition with such techniques as thermographics and vibration analysis, maintenance departments should be able to pre-empt failures and proactively enact measures to avoid downtime. For a review of CBM, see Jardine et al. (2005). This investigative strategy follows the RCM concept, a term coined by Nolan and Heap (1979) and widely disseminated by Moubray (1991).

Design Out Maintenance (DOM) The bottom right-hand section of the grid represents the worst performing assets in both criteria. Major design out projects need to be considered for any machines in this section. An asset in this quadrant is considered not fit for purpose, and hence it should be a candidate for next shut down, overhaul or turnaround activities.

Fixed Time Maintenance (FTM) The FTM sections bordering OTF near the top left-hand section represent "easy" fixed time maintenance. Issues of preventive maintenance include who will carry out the work and when. The FTM sections bordering DOM in the bottom left-hand section are frequently seen as "difficult" FTM. The preventive maintenance issues to be addressed here relate to the actual contents of the jobs.

According to Labib (2004), the above-mentioned strategies are arranged in ascending order with respect to either cost or perceived benefits. Put otherwise, the benefit or cost of maintenance strategies are as follows: OTF $<$ FTM $<$ SLU $<$ CBM $<$ DOM. 


\subsection{Methods of setting thresholds in the DMG}

There are three methods to create partitions between Low, Medium and High Frequency and Downtime. The first method, introduced by Fernandez et al. (2003), is to subtract the lowest values from the highest values, divide by three and multiply by two. Let $h$ be the highest value in the list and $l$ the lowest value in the list. Then

$$
\begin{gathered}
\text { Medium/High Boundary }=h-1 / 3(h) \\
\text { Low/Medium Boundary }=h-2 / 3(h) \\
\text { Low Boundary }=l
\end{gathered}
$$

This method is used when data are homogeneous, and there is an almost equal number of machines in every category. However, if data are not homogeneous, i.e. there is a large discrepancy in the values, the second method is more applicable.

The second method by Tahir et al. (2009) is based on the concept of clustering. The idea is to set partitions at maximum difference by sorting the data in descending order, subtracting the following value from each value in the column and setting the partition at the maximum difference. In other words, let $H$ be the highest value in the list and let $n$ be the equivalent machine. Then

$$
\text { Medium/High Boundary }=\operatorname{Max}\left(H_{n}-H_{n-1}\right)
$$

The same procedure is followed for every partition.

The third method is to use fuzzy sets as proposed in Labib et al. (1998) by setting membership functions for each category based on the judgments of the decision maker. In this method, the idea is to determine membership functions that are either triangular or trapezoidal in order to represent the three categories of Low, Medium and High for both downtime and frequency domains. Fuzzy logic can then be implemented to compute the allocated resources to spend on each strategy. As DMG already contains a set of nine rules (in its $3 \times 3$ matrix), combining this with the membership functions allows the formulation of a fuzzy logic approach based on three steps: fuzzification, rule evaluation and defuzzification. 


\section{Jack-Knife Diagram (JKD)}

The use of the JKD was initiated when Knights (2001) and Knights (2004) applied it to prioritise the unplanned downtime data for electrical failures in a fleet of 13 cable shovels at an open pit copper mine in northern Chile. In a more recent study, Jardine and Tsang (2013) use the JKD to prioritise the downtime of 695 minor components for a fleet of 14 mobile assets in an underground mine. For their part, Pascual et al. (2009) extend the JKD technique of considering three key performance indicators, reliability, maintainability and unavailability, to include explicit economic effects. Finally, Wijaya et al. (2010) visualise failure data by using interval estimation instead of point estimation in a JKD.

Maintenance downtime of a system during a given period of time $T$, due to an intervention code denoted as Downtime $_{j}$, is the product of the number of times, $n_{j}$, that this code occurs and the mean time to repair, $M T T R_{j}$, of the system, expressed as:

$$
\operatorname{Downtime}_{j}(T)=n_{j}(T) \cdot \operatorname{MTTR}_{j}(T)
$$

If all codes are displayed in an $n$ vs MTTR diagram, it is possible to discriminate those codes causing the major downtimes; it is also possible to assess whether the downtimes are related to a high frequency of being out of service. A disadvantage of using equation (5) is that curves of constant downtime, known as iso-downtime curves, are hyperbolae (Pascual et al., 2009); (Knights, 2001); (Knights, 2004). A solution is to take the logarithm of equation (5), written as

$$
\log \left(\operatorname{Downtime}_{j}(T)\right)=\log \left(n_{j}(T)\right)+\log \left(\operatorname{MTTR}_{j}(T)\right)
$$

where $\log$ refers to $\log _{10}$. When an $x-y$ graph is prepared of $\log n_{j}$ against $\log M T T R_{j}$, the isodowntime curves become straight lines with a negative gradient. Logarithmic scatterplots simplify the identification of those failures contributing most to total equipment downtime or cost, whilst permitting the visualisation of the influence of failure frequency and mean downtime.

\subsection{Limit Determination}

When assessing failures, it is necessary to differentiate between Chronic and Acute problems. Threshold values are determined by relative values that depend on the relative magnitude and quantity of data. Repairs requiring lengthy downtime are considered Acute problems. Frequently 
reoccurring failures (i.e. high $n$ ) are considered Chronic problems. By determining the threshold limits, the log scatterplot can be divided into four quadrants as shown in Figure 3. To be consistent with the axes of the DMG, the JKD axes are flipped, with frequency plotted on the yaxis rather than on the $\mathrm{x}$-axis; note that the latter is the more common configuation in the JKD literature. The lower quadrants denote Chronic failures, whilst the right-hand quadrants denote Acute failures. The lower right-hand quadrant is a region of Acute \& Chronic failures.

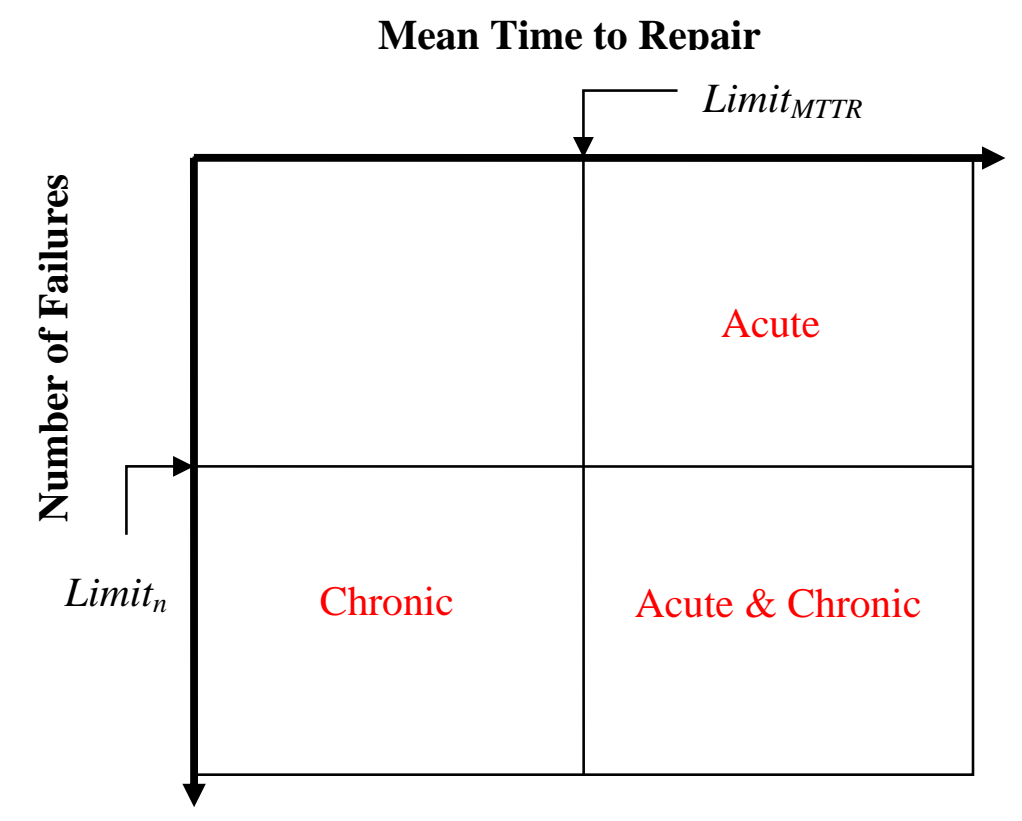

Figure 3. Schematic of JKD showing regions of importance

The total downtime, $D$, caused by unplanned failure is

$$
D_{j}=\sum_{j} \text { Downtime }_{j}
$$

and the total number of failures, $N$, is

$$
N=\sum_{j} n_{j}
$$

When $Q$ represents the number of distinct failure codes used to categorise the repair data, the threshold limit for acute failures can be defined as 


$$
\text { Limit }_{M T T R}=\frac{D}{N}
$$

and the threshold limit for chronic failures becomes

$$
\text { Limit }_{n}=\frac{N}{Q}
$$

\subsection{Downtime priorities and the business cycle}

Maintenance priorities are based on the economic consequences of failures and the associated repair costs. The economic consequences of a failure include the opportunity cost of lost production, the extension of fixed costs such as operator salaries which must be paid irrespective of equipment downtime, the cost of maintaining an increased number of spares as a result of the failure, and the cost of maintaining redundant equipment to mitigate the effects of possible lost production.

In some industries, the economic consequences of plant or equipment downtime for critical equipment outweigh repair and maintenance costs. In such circumstances, it is desirable to prioritise production costs (i.e. equipment availability and reliability) over repair costs. In such a case, where

Cost of lost production >> Repair \& Maintenance Costs, the region of concern is the shaded area in Figure 4. 


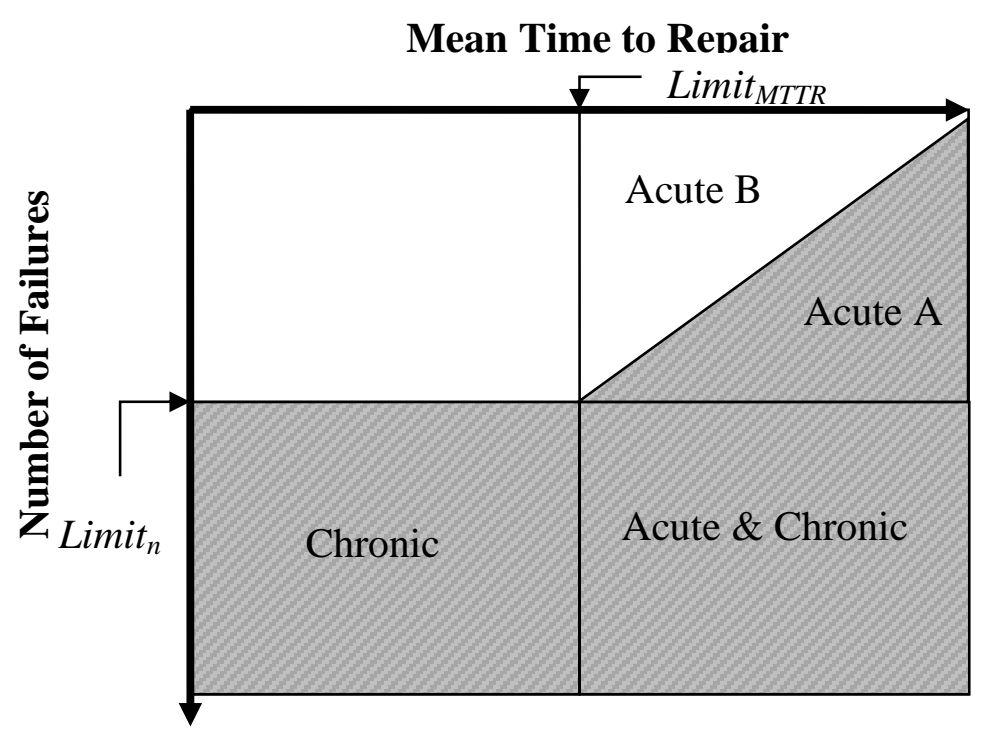

Figure 4. JKD when it is desirable to prioritise production over repair costs.

The availability limit, the line of constant downtime, equals to the product of the two threshold limits calculated in equations (9) and (10) and is expressed as

$$
\left\{\begin{array}{c}
n_{i} \times \text { MTTR }_{i}=\frac{D}{Q} \text { where } 0<n_{i}<\text { Limit }_{n} \\
n_{i}=\text { Limit }_{n} \text { where }_{i} \geq \text { Limit }_{n}
\end{array}\right\}
$$

In the case of commodity price troughs, the cost of production becomes more significant; controlling and reducing O\&M costs will be as important as maintaning equipment and availability. Here, the region of concern is shown in Figure 5, and the expression of the availability limit is

$$
\left\{\begin{array}{c}
\text { MTTR }_{i}=\text { Limit }_{\text {MTTR }} \text { where } 0<n_{i}<\text { Limit }_{n} \\
n_{i} \times \text { MTTR }_{i}=\frac{D}{Q} \text { where }_{i} \geq \text { Limit }_{n}
\end{array}\right\} .
$$




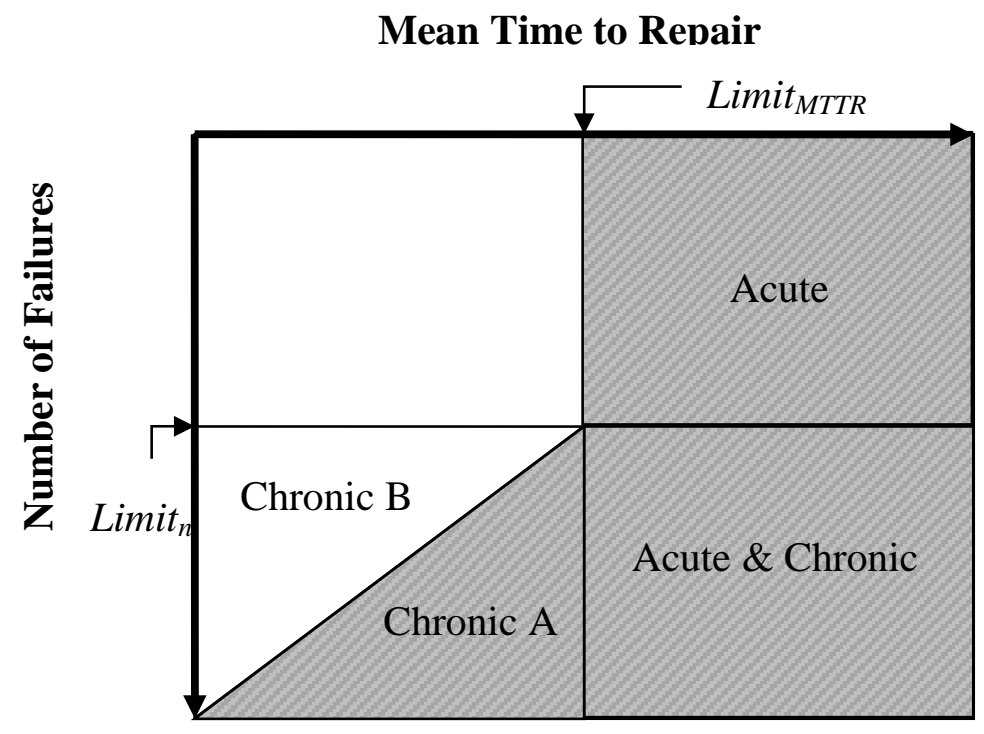

Figure 5. JKD when repair costs are as important as maintaning equipment availability and reliability

According to Knights (2001), greater benefits are obtained by preventing the reoccurence of a single Acute failure than by preventing the reoccurence of a Chronic failure. Hence, all Acute failures should remain in the priority area. The JKD on which this paper focuses is represented by Figure 5. After categorising failure codes, a prioritised list may be developed, similar to the maintenance strategies in the DMG, as follows:

\section{unclassified $<$ Chronic B $<$ Chronic A $<$ Acute $<$ Acute \& Chronic}

Once a prioritised list of failure codes is identified, hypotheses can be made about the possible cause (or causes) of each problem. Root causes of equipment downtime are associated with the following broad categories; equipment design, purchase, storage, installation or start-up, operation and maintenance. Chronic repairs are often associated with design problems, inappropriate operator practices or poor quality control in upstream processes.

An advantage of the JKD over the DMG is that the position of machines/components relative to threshold lines is clear, but this is also due to having only two partitions in the JKD as compared to three partitions in the DMG. According to Knights (2001) and Knights (2004), the JKD can be used as a "trend plot" to visualise the trends in maintenance performance over several time periods. In the first study, Knights (2001) observes improvements in two failure codes over the 
period of the study: Lubrication and Cabin. Unplanned failures to the shovel's lubrication system are classified Chronic in 1997 and 1998 but appear in the unclassified region in 1999. In addition, the total downtime caused by a failure of control in the operator cabin decreases over the period. Two other failure codes go in the opposite direction: unplanned failures in Swing System fall into the Acute region in 1997 but appear in the Acute \& Chronic region in 1998 and 1999. Likewise, failures linked to Alarm increase in both duration and frequency, going from unclassified in 1997 to Chronic in 1998.

\section{Examples}

The three examples given in this section compare the DMG to the JKD.

\subsection{Example 1}

Aslam-Zainudeen and Labib (2011) prepared a DMG using data on a fleet of Class 319 trains from First Capital Connect, a train operating company that operates in the South of the UK. This data will be used to plot the JKD and compare to the DMG. Based on the criteria in Table 1, the DMG and JKD are shown in Figure 6 and Figure 7, respectively. For the JKD limits, based on the data in Table 1 and equations (9) and (10), $D=861$ minutes, $N=56, Q=10$, Limit $_{\text {MTTR }}=$ 15.375, and Limit $_{n}=5.6$. Figure 7 shows the rounded values for the limits.

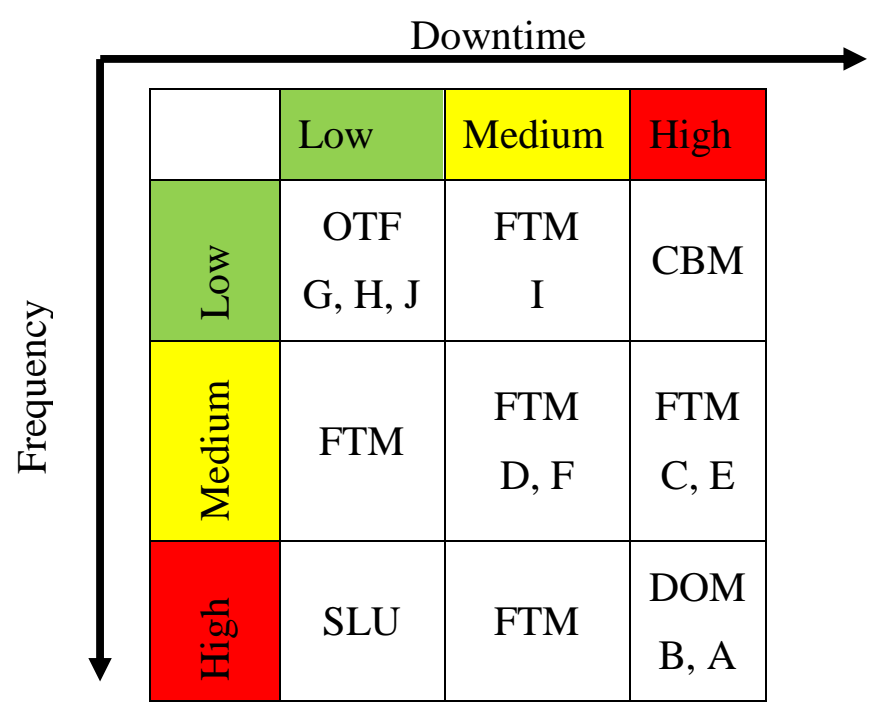

Figure 6. Decision Making Grid for example 1 


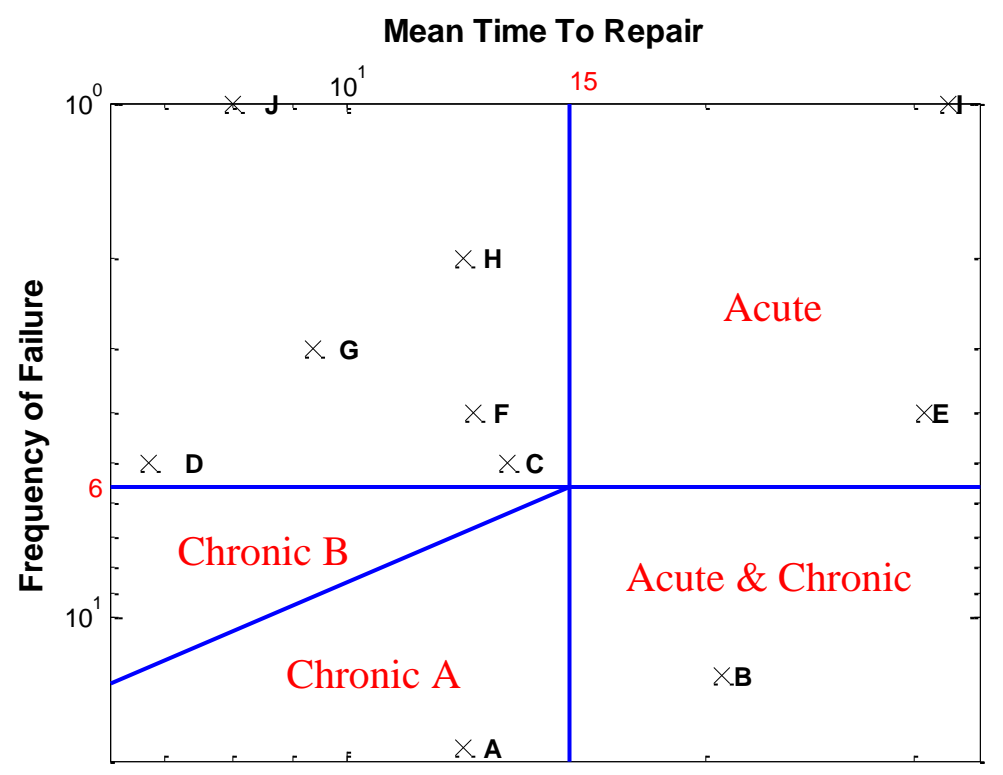

\section{Figure 7. Jack-Knife Diagram for example 1}

In the DMG, machines whose performance is located in the top-right section of the grid, the CBM square, are problematic. They may not break down very often (low frequency) but they represent a major, long-lasting problem when they do (high downtime). A similar region in the JKD is the Acute region. In the DMG, no components appear in the CBM region, but in the JKD, $\mathrm{I}$ and $\mathrm{E}$ appear in the Acute region. For I and E, the JKD seems pessimistic. Upon closer inspection of the location of $\mathrm{I}$ and $\mathrm{E}$ in the JKD relative to the axes, we find $\mathrm{E}$ has a medium Frequency of Failure with high MTTR, which is the same characterisation as in the DMG. However, I has a high MTTR with low Frequency, a pessimistic finding compared to its classification in the DMG.

According to the DMG, the appropriate strategy for A and B is DOM. However, the JKD does not classify A as Acute \& Chronic. OTF components, according to the DMG, are G, H and J, which also fall in the unclassified region of the JKD along with F, C and D, although they are close to the boundaries. The performance of these components will need close monitoring over time. 
Table 1. Criteria Analysis of Class 319 trains for DMG.

\begin{tabular}{|c|c|c|c|c|c|c|c|}
\hline & Frequency & $\begin{array}{l}\text { No. Of } \\
\text { Failures }\end{array}$ & $\begin{array}{l}\text { Criteria } 1 \\
\text { (DMG) }\end{array}$ & & Downtime & $\begin{array}{l}\text { Delay } \\
\text { Minutes }\end{array}$ & $\begin{array}{l}\text { Criteria } 2 \\
\text { (DMG) }\end{array}$ \\
\hline A & Power & 18 & High & B & Doors & 268 & High \\
\hline B & Doors & 13 & High & A & Power & 225 & High \\
\hline $\mathbf{C}$ & Safety Systems & 5 & Medium & $\mathrm{E}$ & Brakes & 122 & High \\
\hline D & Air Systems & 5 & Medium & $\mathrm{C}$ & Safety Systems & 68 & High \\
\hline $\mathbf{E}$ & Brakes & 4 & Medium & $\mathrm{F}$ & $\begin{array}{l}\text { Current Collection } \\
\text { Equipment }\end{array}$ & 51 & Medium \\
\hline $\mathbf{F}$ & $\begin{array}{l}\text { Current Collection } \\
\text { Equipment }\end{array}$ & 4 & Medium & D & Air Systems & 34 & Medium \\
\hline G & $\begin{array}{l}\text { Interior Seats, } \\
\text { Floor and Trims }\end{array}$ & 3 & Low & I & $\begin{array}{l}\text { Train } \\
\text { Communication }\end{array}$ & 32 & Medium \\
\hline H & $\begin{array}{l}\text { Jumpers and } \\
\text { Coupling }\end{array}$ & 2 & Low & G & $\begin{array}{l}\text { Interior Seats, } \\
\text { Floor and Trim }\end{array}$ & 28 & Low \\
\hline I & $\begin{array}{l}\text { Train } \\
\text { Communication }\end{array}$ & 1 & Low & $\mathrm{H}$ & $\begin{array}{l}\text { Jumpers and } \\
\text { Coupling }\end{array}$ & 25 & Low \\
\hline $\mathbf{J}$ & Underframe & 1 & Low & $\mathrm{J}$ & Underframe & 8 & Low \\
\hline
\end{tabular}

\subsection{Example 2}

Table 2 presents unplanned downtime data for electrical failures in a fleet of 13 cable shovels, collected over a one-month period, at an open pit copper mine in northern Chile (Knights, 2001). For DMG mapping, Table 2 allocates codes to Low, Medium and High groups for each criterion according to equations (1) to (3). 
Table 2. Low, Medium and High allocation, for each criterion, for DMG mapping of unplanned shovel electrical downtime

\begin{tabular}{|c|c|c|c|c|c|c|}
\hline Code & Description & $\begin{array}{c}\text { Criterion } 1 \\
\text { (DMG) }\end{array}$ & $\begin{array}{l}\text { Downtime } \\
\text { (Minutes) }\end{array}$ & $\begin{array}{c}\text { Criterion } 2 \\
\text { (DMG) }\end{array}$ & Code & Frequency \\
\hline '1' & Electrical Inspections & High & 1015 & High & '11' & 36 \\
\hline '2' & Damaged feeder cable & High & 785 & High & '1' & 30 \\
\hline '11' & Motor overtemperature & High & 745 & High & '3' & 27 \\
\hline '3' & $\begin{array}{c}\text { Change of substation or } \\
\text { shovel move }\end{array}$ & High & 690 & High & '9' & 26 \\
\hline$' 10 '$ & Overload relay & High & 685 & High & '10' & 23 \\
\hline '7' & Auxiliary motors & Medium & 600 & Medium & '5' & 21 \\
\hline$' 12 '$ & Earth faults & Medium & 575 & Medium & '2' & 15 \\
\hline '8' & Main motors & Medium & 555 & Medium & '4' & 15 \\
\hline '5' & Power cuts to substations & Medium & 395 & Medium & '7' & 13 \\
\hline$' 15 '$ & Air compressor & Medium & 355 & Medium & ' 8 ' & 12 \\
\hline '6' & Rope limit protection & Low & 277 & Low & '6' & 10 \\
\hline '9' & Lighting system & Low & 240 & Low & '13' & 9 \\
\hline '4' & Coupling repairs or checks & Low & 225 & Low & '15' & 8 \\
\hline$' 17^{\prime}$ & Overcurrent faults & Low & 220 & Low & '12' & 7 \\
\hline '14' & Control system & Low & 165 & Low & '14' & 7 \\
\hline$' 16 '$ & Operator controls & Low & 155 & Low & '17' & 6 \\
\hline$' 13 '$ & Miscellaneous & Low & 115 & Low & '16' & 5 \\
\hline
\end{tabular}




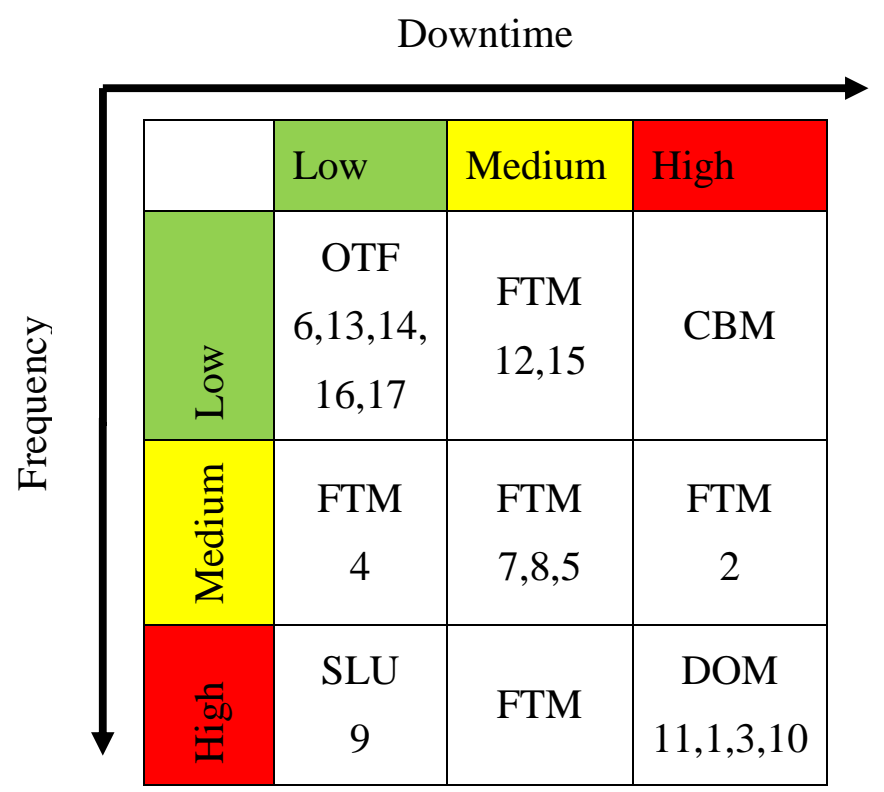

Figure 8. Decision Making Grid for example 2

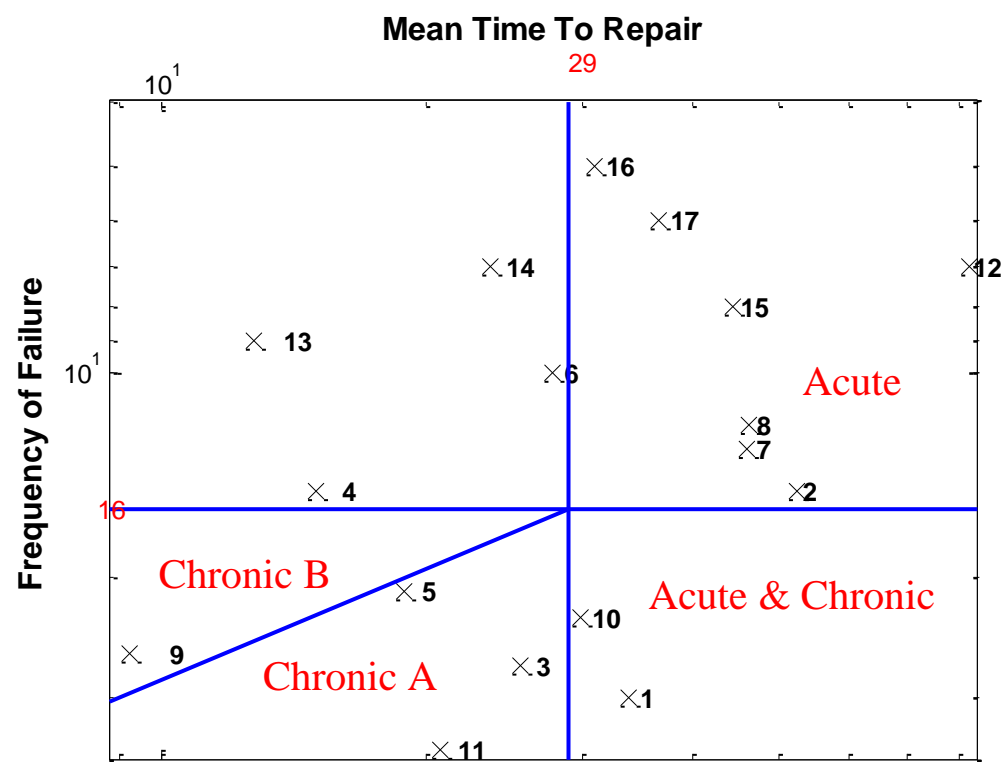

Figure 9. Jack-Knife Diagram for example 2.

Components in the OTF section in the DMG and the unclassified region in the JKD will be discussed first. The diagrams' overlapping codes are 6, 13 and 14. In the DMG, 4 falls in the mid 
left-hand section of the grid but is unclassified in the JKD (although very close to Limit $_{n}$ border); in the JKD, 17 appears in the Acute region but is in the OTF grid in the DMG. The advantage of the JKD here is the availability of a visual representation of the proximity of a failure code to either the Limit or Limit

A seemingly big difference between the DMG and the JKD is the classification of 17 . In the DMG, 17 is of little concern and should operate as normal to failure. However, the JKD classifies this component as Acute. If closer attention is paid to the axes of the JKD, the frequency of failure for component 17 is low, and its MTTR is midway. For 17, the JKD is pessimistic.

Now, looking at the DOM section of the DMG and the Acute \& Chronic grid of the JKD, the overlappying codes are 1 and 10 respectively. In the DMG, components 3 and 11 also fall in the DOM grid but appear only in the Chronic A region of the JKD. In this case, the DMG is pessimistic. In the JKD, 3 is closer to the Limit ${ }_{\text {MTTR }}$ border and should be monitored closely over time. Again, the advantage of the JKD is that we can see how far components are from the borders.

\subsection{Example 3}

Table 3 provides a list of scheduled and unscheduled breakdowns for a Geomembrane plant in 2007. Figures 10 and 11 show the corresponding DMG and JKD plots for the data in Table 3.

Downtime

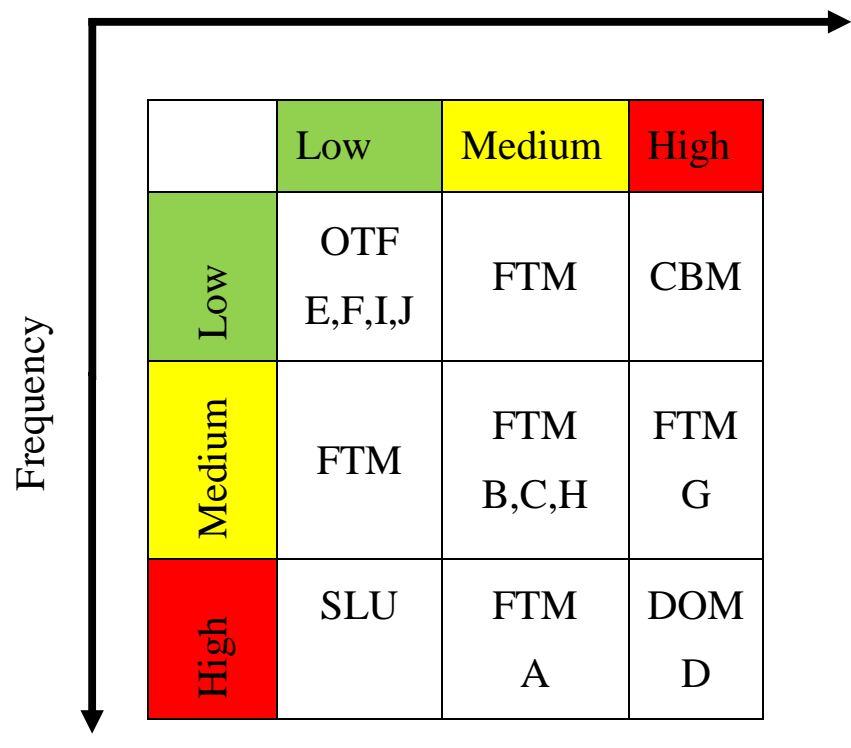


Figure 10. Decision Making Grid for example 3.

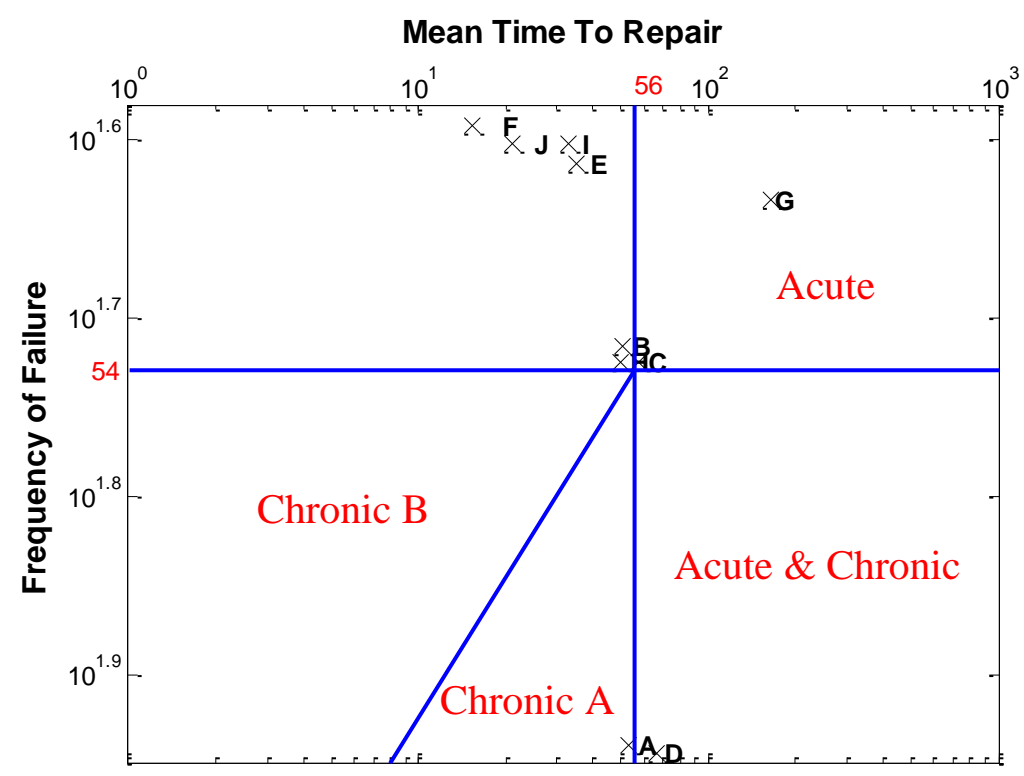

Figure 11. Jack-Knife Diagram for example 3.

In Figure 10, D falls in the DOM section of the grid, with E, F, I and $\mathrm{J}$ in the $O T F$ section. In the JKD, D falls in the Acute \& Chronic region, a classification similar to that of the DMG. No components require CBM according to the DMG. G is classified as Acute in the DMG, and A falls in the Chronic A region. A falls in the FTM region in the DMG (medium MTTR and high Frequency of Failure) which, upon comparison with its position in the JKD, is similarly classified. The OTF components of the DMG are E, F, I and J, which fall in the unclassified region in the JKD along with $\mathrm{B}$ and $\mathrm{H}$, although they are very close to limit lines. Although $\mathrm{H}$ is classified as Acute in the JKD, it too falls very close to the Limit ${ }_{M T T R}$ border. Observation of the positions of $\mathrm{B}, \mathrm{C}$ and $\mathrm{H}$ relative to the JKD axes reveals their locations mimic the results shown in the DMG of medium frequency and medium downtime. 
Table 3. High, Medium and Low classification for DMG

\begin{tabular}{|c|c|c|c|c|c|}
\hline Code & Description & Frequency & $\begin{array}{c}\text { Criterion } 1 \\
\text { (DMG) }\end{array}$ & Code & $\begin{array}{cc}\text { Downtime } & \text { Criterion 2 } \\
\text { (Minutes) } & \text { (DMG) }\end{array}$ \\
\hline
\end{tabular}

\begin{tabular}{|c|c|c|c|c|c|c|}
\hline 'D' & Flat T-Die $2400 \mathrm{~mm}$ & 88 & High & 'G' & 7080 & High \\
\hline 'A' & Vacuum loading system & 87 & High & 'D' & 5820 & High \\
\hline 'C' & $\begin{array}{l}\text { (i) Gear box (single helical } \\
\text { gear type) Output speed } \\
=58 \mathrm{RPM} \\
\text { (ii) Extruder ( } 120 \varnothing \text { screw) } \\
\text { (iii)DC Driving motor } 55 \mathrm{~kW} \\
\text { and } 1150 \mathrm{RPM}\end{array}$ & 53 & Medium & 'A' & 4620 & Medium \\
\hline 'H' & Cooling unit & 53 & Medium & 'C' & 3060 & Medium \\
\hline 'B' & $\begin{array}{l}\text { (i) Gear box (single helical } \\
\text { gear type) Output speed } \\
=58 \mathrm{RPM} \\
\text { (ii) Extruder ( } 120 \varnothing \text { screw) } \\
\text { (iii)DC Driving motor } 75 \mathrm{~kW} \\
\text { and } 1150 \mathrm{RPM}\end{array}$ & 52 & Medium & 'B' & 2640 & Medium \\
\hline 'G' & Winding system & 43 & Medium & 'H' & 2640 & Medium \\
\hline 'E' & $\begin{array}{l}\text { Emboss, polishing and } \\
\text { cooling rolls system }\end{array}$ & 41 & Low & 'E' & 1440 & Low \\
\hline 'I' & Air compressor & 40 & Low & 'I' & 1320 & Low \\
\hline 'J' & Overhead crane & 40 & Low & 'J' & 840 & Low \\
\hline 'F' & Take-up system & 39 & Low & 'F' & 600 & Low \\
\hline
\end{tabular}




\subsection{Discussion}

This paper discusses two tools for allocating maintenance strategies while prioritising machine downtime according to two important downtime criteria: frequency and length of downtime. The DMG and the JKD allocate appropriate maintenance strategies depending on the location of the machines within the diagrams. To this point, the JKD has been used to prioritise machine downtime, whilst the DMG has been used to allocate maintenance strategies.

Within the DMG, each maintenance strategy can be the responsibility of a team in the maintenance department (function). For example, one team can be responsible for sustaining and auditing best practice for machines in the OTF region, with another team responsible for preventive maintenance of machines in the FTM region. There can be teams for both RCM and CBM investigative approaches, a team for TPM and upgrading maintenance skills strategies, and a team for major overhauls and redesign projects of machines in the DOM quadrant. In short, the DMG model can be used as a framework for the allocation of machines to various maintenance teams and the performance monitoring of those machines by the respective teams.

Using the DMG as a comparative guide, the case studies show how the JKD allocates maintenance strategies. From the examples presented, it is not obvious which tool is pessimistic. In the cases where the JKD seems pessimistic, upon closer inspection of the location of the components relative to the axes of the JKD, we find their position is similar to that shown in the DMG. The difference is that the JKD contains four sections to allocate four maintenance strategies, whilst the DMG contains nine and can allocate five. In the JKD, there is no clear section to allocate Fixed Time Maintenance (FTM).

The JKD's advantage over the DMG lies in its scaled axes. The location of machines relative to limit lines is measurable. Therefore, if no maintenance action is taken, the user (maintenance technician) has a good idea of which region the machine/failure code might fall into next. Although not obvious from the case studies presented, the JKD can be used in "trend plots" to monitor the movement of machine performance over several time periods. Although this is possible in the DMG, the DMG is almost "binary" in nature for each cell; either a machine falls in one of the sections of the grid or it does not. It is not obvious to see how far a machine is from the limits, unless a method such as fuzzy logic is incorporated, as reported in Labib et al, (1998), 
Sudiarso and Labib (2002), and Yniarto, and Labib (2005). Another advantage of the JKD over the DMG is its mechanism to find the threshold limits.

\subsection{Conclusion}

To be competitive, maintenance organisations must provide timely delivery of quality products. Adopting the right maintenance policy is key to achieving this objective; it reduces costs by minimising downtime and leads to improved quality and productivity. A practical maintenance policy selection method is preferable, but the various maintenance strategies are incomparable. The findings of this paper suggest the DMG and JKD are suitable tools to allocate appropriate maintenance strategies based on two downtime criteria: frequency and length of downtime.

It is clear from this study that both the DMG and JKD have pros and cons and, as such, there is no 'winner'. However, it is hoped that such work will encourage further research to integrate the methods, validate them, or develop more innovative approaches towards criticality analysis and the selection of appropriate maintenance strategies.

\section{References}

1. Al-Najjar, B. and Alsyouf, I. (2003), "Selecting the most efficient maintenance approach using fuzzy multiple criteria decision making", International Journal of Production Economics, Vol. 84, pp. 85-100.

2. Alsyouf, I. (2009), "Maintenance practices in Swedish industries: Survey results", International Journal of Production Economics, Vol. 121, pp. 212-223.

3. Aslam-Zainudeen, N. and Labib, A. (2011), "Practical Application of the Decision Making Grid (DMG)", Journal of Quality in Maintenance Engineering, Vol. 17, No. 2, pp 138-1491.

4. Bashiri, M., Badri, H. and Hejazi, T.H. (2011), "Selecting optimum maintenance strategy by fuzzy interactive linear assignment method", Applied Mathematical Modelling, Vol. 35, pp. $152-164$.

\footnotetext{
${ }^{1}$ Received the 2012 "Highly Commended Award" from the Emerald Literati Network.
} 
5. Bevilacqua, M. and Braglia, M. (2000), "The analytic hierarchy process applied to maintenance strategy selection", Reliability Engineering and System Safety, Vol. 70, pp. 7183.

6. BSI Standards 13306, Maintenance Terminology Standards, BS EN, 2001.

7. Burhanuddin, M. A., Ahmad, A. R. and Desa, M. I. (2007), "An Application of Decision Making Grid to Improve Maintenance Strategies in Small and Medium Industries", in Proceedings of the 2nd IEEE Conference on Industrial Electronics \& Applications, pp. 455460.

8. Chakhar, S., Ishizaka, A., Labib, A., Saad, I. (2016), "Dominance-based rough set approach for group decisions", European Journal of Operational Research, Vol. 251, Vol. 1, pp. 206224.

9. Cho, I. D. and Parlar, M. (1991), "A survey of maintenance models for multi-unit systems", European Journal of Operational Research, Vol. 51, pp. 1-23.

10. Fernandez, O., Labib, A.W., Walmsley , R. and Petty D. J. (2003), “A Decision Support Maintenance Management System: Development and Implementation", International Journal of Quality and Reliability Management, Vol. 20, No. 8, pp. 965-979.

11. Hartmann, E. H. (1992), Successfully Installing TPM in a Non-Japanese Plant, TPM Press Inc., New York.

12. Hu, Q., Chakhar, S., Siraj, S., Labib, A., (accepted, 2017), Spare parts classification in industrial manufacturing using the dominance-based rough set approach, European Journal of Operational Research.

13. Jardine, A. K. S. and Tsang, A. H. C. (2013), Maintenance, Replacement, and Reliability Theory and Applications, $2^{\text {nd }}$ Edition, CRC Press Taylor \& Francis Group, Boca Raton, FL.

14. Jardine, A. K. S., Lin, D., Banjevic, D. (2005), "A review on machinery diagnostics and prognostics implementing condition-based maintenance", Mechanical Systems and Signal Processing, Vol 20, No. 7, pp. 1483-1510.

15. Kaplan, R. S. and Norton, D. P. (1992), “The Balanced Scorecard: Measures That Drive Performance", Harvard Business Review, Jan. - Feb, pp. 71 - 79.

16. Kumar, U., Galar, D., Parida, A. and Stenstrom, C. (2013), "Maintenance performance metrics: a state-of-the-art review", Journal of Quality in Maintenance Engineering, Vol. 19 No. 3, pp. 233-277. 
17. Knights P.F. (2001), "Rethinking Pareto Analysis: Maintenance Applications of Logarithmic Scatterplots", Journal of Quality in Maintenance Engineering, Vo. 7, No. 4, pp. 252-263.

18. Knights, P.F. (2004), "Downtime Priorities, Jack-Knife Diagrams and the Business Cycle", Maintenance Journal, Vol. 17, No. 2, pp. 14-21.

19. Kobbacy, K. A. H., Proudlove, N. C. and Harper, M. A. (1995), "Towards an intelligent maintenance optimization system", Journal of the Operational Research Society, Vol. 46, No. 7, pp. 831-853.

20. Labib, A.W. (1996), An Interactive and Appropriate Productive Maintenance, $\mathrm{PhD}$ thesis, University of Birmingham, Birmingham, U. K.

21. Labib, A.W., O'Connor, R.F., and Williams, G.B. (1996), "Formulation of an appropriate maintenance strategy using multiple criteria decision making", Maintenance Journal, Vol. 11, No. 2, pp. 14-21.

22. Labib, A.W., O’Connor, R.F., and Williams, G.B. (1997), "Deriving A Maintenance Strategy Through the Application of a Multiple Criteria Decision Making Methodology", Fandel, G. And Gal, T., Lecture Notes in Economics and Mathematical Systems, No. 448; SpringerVerlag, Berlin, Germany, pp. 481-490.

23. Labib, A.W., O'Connor, R.F. and Williams, G.B. (1998a), "An Effective Maintenance System Using the Analytic Hierarchy Process", Journal of Integrated Manufacturing Systems, Vol. 9, No. 2. pp 87-98.

24. Labib, A.W., Williams. G.B. and O'Connor, R.F. (1998b), "An Intelligent Maintenance Model (System): An Application of A.H.P. and a Fuzzy Logic Rule-Based Controller", Journal of Operational Research Society; Vol 9, No. 7.

25. Labib, A.W. (1998), "World Class Maintenance Using a Computerised Maintenance Management System", Journal of Quality in Maintenance Engineering, Vol. 4, No. 1, pp 6675.

26. Labib, A.W., Computerised Maintenance Management Systems (CMMSs): A black hole or a black box?, Journal of Maintenance \& Asset Management, ISSN 0952-2110, Vol 18 No3, pp 16-21, 2003.

27. Labib, A.W. (2004), "A decision analysis model for maintenance policy selection using a CMMS", Journal of Quality in Maintenance Engineering, Vol 10, No 3, pp. 191-202 
28. Labib, A. (2014), Learning From Failures: Decision Analysis of Major Disasters, Butterworth-Heinemann, Oxford.

29. Lazakis, I. and Olcer, A. (2015), "Selection of the best maintenance approach in the maritime industry under fuzzy multiple attributive group decision-making environment", in Proceedings of the Institution of Mechanical Engineers, Part M: Journal of Engineering for the Maritime Environment, pp. 1-13.

30. Mc Leod, J., Bazuik, P., Calvo, R., and Rivera, S. (2015), "Failures profiles for maintenance in industrial facilities", in Proceedings of the World Congress on Engineering, Vol II, WCE, London, UK.

31. Moubray, J. (1997), Reliability Centred Maintenance II ,Butterworth Heinemann, Oxford, U.K.

32. Muchiri, P., Pintelon, L. and Martin, H. (2010), "Empirical Analysis of Maintenance Performance Measurement in Belgian Industries", International Journal of Production Research, Vol. 48, No. 20, pp. 5905-5924.

33. Nakajima, S., (1988), Introduction to TPM: Total Productive Maintenance, Productivity Press, Cambridge, U.K.

34. Nakhjavani, A., Mollaverdi, N., Rafiei, F. (2014), "Hybrid approach of ANP and DEMATEL for selecting optimal maintenance stategy: a case study in the phrmaceutical industry", International Journal of Applied Research on Industrial Engineering, Vol. 1, No. 4, pp. 230249.

35. Neely, A., Gregory, M. and Platts, K. (2005), "Performance Measurement System Design: Literature Review and Research Agenda", International Journal of Operations \& Production Management, Vol. 25, No. 12, pp. 1228-1263.

36. Nolan, F. and Heap, H. (1978), Reliability Centred Maintenance, United Air Lines Inc., San Francisco, CA, U.S.A.

37. Oyarbide-Zubillaga, A., Goti, A., and Sanchez, A. (2008), "Preventive maintenance optimisation of multi-equipment manufacturing systems by combining discrete event simulation and multi-objective evolutionary algorithms", Production Planning \& Control, Vol. 19, No. 4, pp. 342-355. 
38. Parida, A. and Chattopadhyay, G. (2007), "Development of a multi-criteria hierarchical frameworkfor maintenance performance measurement (MPM)", Journal of Quality in Maintenance Engineering, Vol.13, pp. 241-258.

39. Pascual, R., Del Castillo, G., Louit, D. and Knights, P. (2009), "Business-Oriented Prioritization: A Novel Graphical Technique”, Reliability Engineering and System Safety, Vol. 94, pp. 1308-1313.

40. Picciolo, G., Galli, F., Biamonti, A. and Magni, P. (2008), "Determining the maximum periodic inspection interval for medium voltage motors using a Markov model", Production Planning \& Control, Vol. 19, No. 4, pp. 356-364.

41. Pinjala, S. K., Pintelon, L., Vereecke, A. (2006), "An empirical investigation on the relationship between business and maintenance strategies", International Journal of Production Economics, Vol. 104, pp. 214-229.

42. Pourjavad, E. and Shirouyehzad, H. (2014), “Analysing maintenance strategies by FANP considering RAM criteria: a case study", International Journal of Logistics Systems and Management, Vol. 8, No. 3, pp. 302-321.

43. Shahin, A. and Attarpour, M. R. (2011), "Developing Decision Making Grid for Maintenance Policy Making Based on Estimated Range of Overall Equipment Effectiveness", Modern Applied Science, Vol. 5, No. 6, pp. 86-97.

44. Simoes, J. M., Gomes, C. F. and Yasin, M. M. (2011), "Reviews and Case Studies A Literature Review Of Maintenance Performance Measurement: A Conceptual Framework And Directions For Future Research”, Journal Of Quality In Maintenance Engineering, Vol. 17 No. 2, pp. 116-137

45. Sudiarso, A and A.W. Labib (2002), A Fuzzy Logic Approach to an Integrated Maintenance / Production Scheduling Algorithm, International Journal of Production Research (IJPR), Vol 40, No 13 pp 3121-3138.

46. Swanson, L. (2001), "Linking maintenance strategies to performance”, International Journal of Production Economics, Vol. 70, pp. 237-244.

47. Tahir, Z., Prabuwono, A. S., Burhanuddin, M. A. and Akbar, H. (2008), "Maintenance Decision Support Fuzzy System in Small and Medium Industries using Decision Making Grid", in Intenational IEEE Conference on Advanced Computer Theory and Engineering 20- 
22 December 2008, in Phuket, Thaniland, IEEE Computer Society, Los Alamitos, CA, pp. 680-684.

48. Tahir, Z, Burhanuddin, M. A., Ahmad, A. R., Halawani, S.M., and Arifi, F. (2009), "Improvement of Decision Making Grid Model for Maintenance Management in Small and Medium Industries", in IEEE Explorer, Proceedings of International Conference on Industrial and Information Systems (ICIIS), pp. $598-603$.

49. Tahir, Z., Ahmad, A., Aboobaidder, B., and Koayashi, S. (2014), "Using genetic algorithm to bridge decision making grid data gaps in small and medium industries", in Makassar International Conference on Electrical Engineering and Informatics (MICEEI), IEEE, Indonesia.

50. Vanneste, S. G. and van Wassenhove, L. N. (1995), “An integrated and structured approach to improve maintenance", European Journal of Operational Research, Vol. 82, No. 2, pp. 241-257.

51. Wang, L., Chu, J., and Wu, J. (2007), "Selection of optimum maintenance strategies based on a fuzzy analytic hierarchy process", International Journal of Production Economics, Vol. 107, pp. 151-161.

52. Wijaya, A. R., Lundber, J. and Kumar, U. (2010), "Graphical Method for Visualization of Machine's Downtime (Case study of Scaling Machine)", The $1^{\text {st }}$ International Workshop and Congress on eMaintenance, Lulea, Sweden, pp. 22-24.

53. Willmott, P. (1994), Total Productive Maintenance. The Western Way, Butterworth Heinemann Ltd.,Oxford, U. K.

54. Yuniarto, N., and A.W. Labib (2005), Optimal Control of an Unreliable Machine Using Fuzzy Logic Control: From Design to Implementation, International Journal of Production Research, (IJPR), , Vol. 43, No. 21, pp 4509-4537. 